\title{
Quantifying the Differences in Geometry and Size Distributions of Buildings Within Cities
}

\author{
Nahid Mohajeri • Agust Gudmundsson
}

Published online: 28 May 2014

(C) Kim Williams Books, Turin 2014

\begin{abstract}
There have been many studies on the spatial configuration of cities, but few attempts to quantify the difference in building patterns between the old and new parts of cities. This may be partly attributable to lack of suitable study methods. This paper presents a new application of statistical methods for quantifying the geometric difference between different parts of a city using, as a case study, the old (historical) and new parts of the city of Yazd in Iran. We measured 341 edge lengths of 4 bazaars, 302 edge lengths of 5 mosques and tombs, and 239 edge lengths of 3 schools. We also measured 6,804 edge lengths and the areas of 1,243 well-preserved courtyard houses in the old part and 4,948 edge lengths and the areas of 1,237 houses in the new part of the city. In the old part, all edge-length and house-area frequency distributions, to a first approximation, follow power laws, indicating that there are many small and very few large buildings. By contrast, in the new part the edge-length and house-area frequency distributions follow bimodal (two-peak) distributions. The calculated entropies (measures of dispersion) of the house edge lengths and areas in the old part are much higher than of those in the new part and provide a clear, quantitative measure of the geometric differences between the builtup structures of old and the new parts of the cities. The power-law distributions in the old part indicate a gradual and continuous variation in sizes of edge lengths and house areas, whereas the bimodal distributions in the new part indicate abrupt (discontinuous) changes in the edge lengths and house areas. The entropy results imply that the size distributions of houses in the old part are more dispersed than those in the new part, indicating more interconnected structures in the old part of the
\end{abstract}

\footnotetext{
N. Mohajeri $(\bowtie)$

Solar Energy and Building Physics Laboratory (LESO-PB),

Ecole Polytechnique Fédérale de Lausanne (EPFL), 1015 Lausanne, Switzerland e-mail: nahid.mohajeri@epfl.ch
}

\footnotetext{
A. Gudmundsson

Department of Earth Sciences, Royal Holloway University of London, London, UK e-mail: a.gudmundsson@es.rhul.ac.uk
} 
city. The results also demonstrate quantitatively that there is a lack of coherence between the structures of old and new parts of city.

Keywords Urban design - Spatial configuration - Building geometries ·

Size distribution - Statistical analysis · Entropy

\section{Introduction}

There have been many studies of the geometric patterns of cities. Some use fractal analysis (e.g., Batty and Longley 1994; Bovill 1996; Salingaros and West 1999; Salingaros 2005; Liang et al. 2013) while others focus on the relation between the geometric patterns and energy (Kasmaii 1983; Steemers et al. 1998; Ratti et al. 2003; Johansson 2006; Kämpf et al. 2009; Fuller and Crawford 2011). City morphology, socio-cultural patterns, and associated landscape architecture (Clark and Costello 1973; Bonine 1979; Kostof 1991; Kheirabadi 2000; Memarian and Brown 2003; Hu 2008; Habib et al. 2012), street patterns (Marshall 2005; Scellato et al. 2006; Mohajeri and Gudmundsson 2012; Gudmundsson and Mohajeri 2013), and size distributions (Batty et al. 2008; Mohajeri et al. 2013) have also been the subject of recent studies.

Many cities contain parts that have widely different built-up geometric patterns. A good example is the city of Yazd, located in central Iran (Fig. 1). Here the contrast in geometric pattern is between the old historical part and the new (more recent) part of the city. The built-up structures in the old (historical) part vary in size, are irregular in shape, and have curved pathways and streets. The old part developed gradually and has a well-interconnected structure (Kheirabadi 2000; Memarian and Brown 2003). The new part has developed largely independently of the old part and is geometrically different from the old, historical part (Karimi 2000; Zanganeh Shahraki et al. 2011; Sharifi and Murayama 2013).

Yazd is particularly suitable for this kind of study because a large fraction of its old part has maintained its original structure for thousands of years, whereas a large fraction of the new part dates from the last decades. The city has expanded

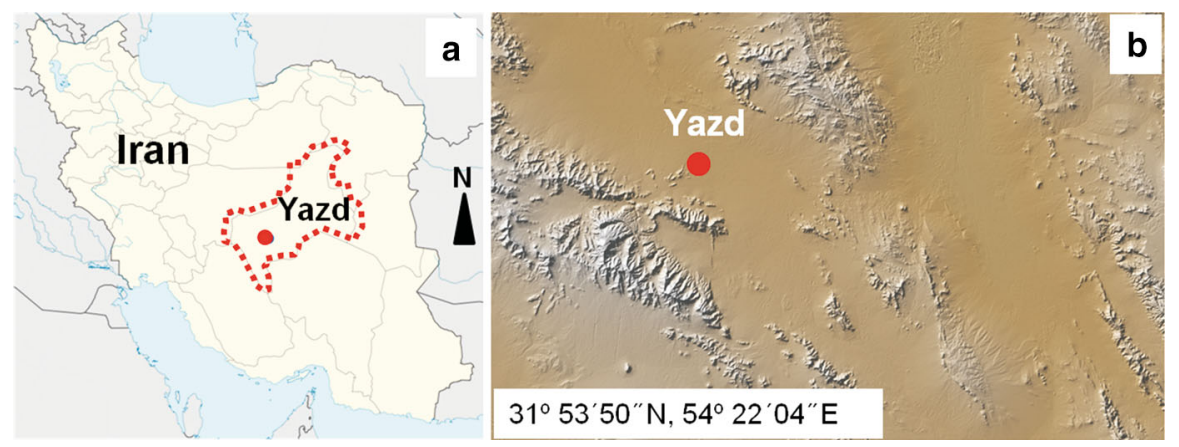

Fig. 1 a Geographical location of the city of Yazd in Iran (Wikipedia). b The location of the city of Yazd in relation to the surrounding terrain (http://www.geomapapp.org) 
enormously in the past decades: in 1975 its population was about 136,000, whereas in 2009 its population was about 465,000 (Iranian Statistical Centre 2009).

A quantitative study of Yazd is important in that it throws light on the textural development of cities that (a) are of a medium size (population between 100,000 and 500,000), (b) have had very rapid recent urban growth, (c) are located in hotarid regions, and (d) have new parts that have developed largely independently of, and are geometrically different from, the old (historical) parts. Examples of point (d) include the cities of Tunis in Tunisia, Sana'a in Yemen, and Bukhara in Uzbekistan (http://whc.unesco.org).

While it is commonly clear from aerial images that the new and old parts of a city do not fit smoothly together as regards their geometric patterns, little attempt has been made to quantify the geometric difference between the parts. One reason for so little work in this direction may be the lack of suitable methods for quantifying the textural difference. The principal aim of this paper is to show, using the example of Yazd (Fig. 1a), how geometric differences between parts of a city can be quantified by methods that have not, previously, been used for such a study. The focus is on detailed measurements of the edge lengths and the areas (sizes) of various built structures, so as to detect their underlying order and to compare the geometric patterns of the old part with those of the new part of the city. We select edge lengths and areas for the present study because these are easily and accurately measureable quantities (through AutoCad/GIS), but in a further development of the approach used here other parameters could be used, such as, for example, the heights of the buildings. Using calculated entropies, length ranges (differences between the maximum and the minimum length), and the scaling-exponents of the size distributions, we are able to quantify the geometric patterns of the old and the new parts of the city of Yazd and show how they are fundamentally different.

\section{Geographical and Historical Background of Yazd}

The city of Yazd $\left(31^{\circ} 54^{\prime} \mathrm{N}, 54^{\circ} 22^{\prime} \mathrm{E}\right)$ is located in the eastern central part of Iran, the heart of the main desert area in the country, in a wide dry valley between the southwestern and northeastern parts of the mountains of Shirkooh (Fig. 1b). The maximum daily summer temperature commonly reaches $40{ }^{\circ} \mathrm{C}$, during which time there is hardly any rain at all. The total annual rainfall is $50-60 \mathrm{~mm}$. The city climate is characterised by hot and very dry summers and cooler and dry winters.

Because of the shortage of surface water, Yazd depends very much on the groundwater channelled through a system of qanats. The system is based on underground tunnels or galleries to extract groundwater and has been described as the greatest contribution by Persians to the science of hydraulics (Lambton 1992; English 1998). Because of the difficult climatic conditions and the shortage of water, agriculture is not of primary importance for present-day Yazd, which is primarily a trading and industrial city.

The historical part of the city is more than 3000 years old and regarded as the second oldest continuously inhabited city in the world after the city of Venice in Italy (http://whc.unesco.org). It is culturally, historically, and architecturally a 

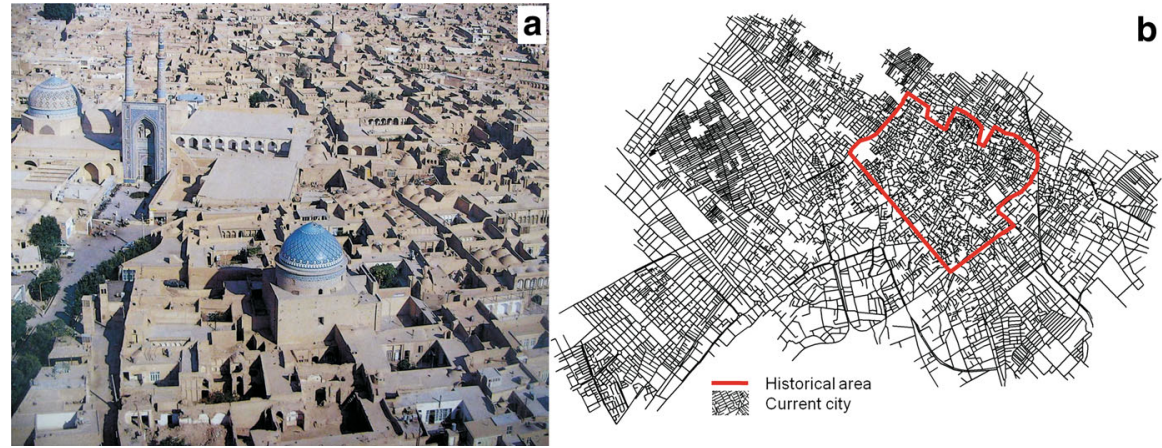

Fig. 2 a Birdseye-view of the historical city of Yazd (Yazd Cultural Heritage and Tourism Organization 2005). b The whole city of Yazd (GIS street map of the city) with the historical area marked by a red line

remarkable city. The present-day city is of medium size, with a population about 465,000 in 2009 (Iranian Statistical Centre 2009).

The old (historical) part of Yazd is very compact and divided into several neighbourhoods. Each neighbourhood is like a small town, containing nearly all the main structures of a city centre, such as a bazaar, a mosque, a religious school, as well as underground water storage (ab-anbar) and the residential quarters around them. The old part has also a city centre which consists of the Jameh Mosque, a tomb, a bazaar, a religious school, a public bath, and residential quarters (Habibi 1998; Tavasoli 1989, 2001; Sharifi and Murayama 2013). The location of the old part within the modern city is shown in Fig. 2.

The courtyard houses, the most common built-up structures of the old part, vary considerably in form and size. These types of houses date back to the earliest settlements in the Middle East (Lampl 1968; Lavas 1974; Bonine 1979; Memarian and Brown 2003). Most houses are single storey (one floor) and are densely clustered along narrow and curved pathways or streets. The traditional courtyard houses are inward looking with a courtyard in the centre (Fig. 3), arranged so that they are side by side and back to back (Tavasoli 1989, 2001; Memarian and Brown 2003). The courtyard houses have thick walls, commonly 400-800 mm thick (Kasmaii 1983; Memarian and Brown 2003; Tavasoli 1989, 2001). Commercial activity was traditionally confined to bazaars, often arranged by trade, with stalls opening directly onto the streets, covered by brick vaults or domes. Open public spaces are mainly associated with mosques, tombs, and schools for the creation of several small squares and the widening of some alleys. The new part of the city, by contrast, has a completely different structure. Buildings are outward looking and the streets, which are designed mainly for vehicles, are wide and follow a grid pattern, thereby forming a regular network (cf. Fig. 2b).

\section{Data}

We analysed the geometric properties of built-up areas in both the old and the new parts of the city using a two-dimensional Auto-CAD/GIS model of Yazd based on datasets from the National Cartographic Centre of Iran (2005). This is a digital 
model of all the buildings in the selected sample areas, namely an area of $793,734 \mathrm{~m}^{2}$ (about $0.80 \mathrm{~km}^{2}$ ) from the old part and an area of 972,418 $\mathrm{m}^{2}$ (about $0.97 \mathrm{~km}^{2}$ ) from the new part of the city. To digitalise the data we used the AutoCAD maps of Yazd and converted them into GIS-shape files. The Auto-CAD dataset of Yazd was generated in 2003 by the National Cartographic Centre of Iran using a UTM projection system and WGS-84 datum for the entire urban area of Yazd based on aerial photography at the scale 1:4,000.

Aerial images (National Cartographic Centre of Iran 2005) and historical maps (Tavasoli 1989, 2001; Ministry of Housing and Urban Development 2005) of Yazd were used to identify the main structures and their geometric patterns in the selected study parts of the city (Fig. 3). All the data used here on the old part are from the oldest part of the historical area, outlined in blue in Fig. 3. A considerable fraction of this part has been largely preserved for thousands of years. The historical city, located within the city walls (in blue in Fig. 3), is partly from the Islamic period, that is, younger than the 7th century. However, all of it is older than the 14th century (Kalantari and Hataminejad 2006).

We define the edge length of a building as the straight-line length from one corner along its walls to the next corner (Fig. 4). Using this definition, we measured the edge lengths of many specific buildings in the old part (mosques, bazaars, and schools) as

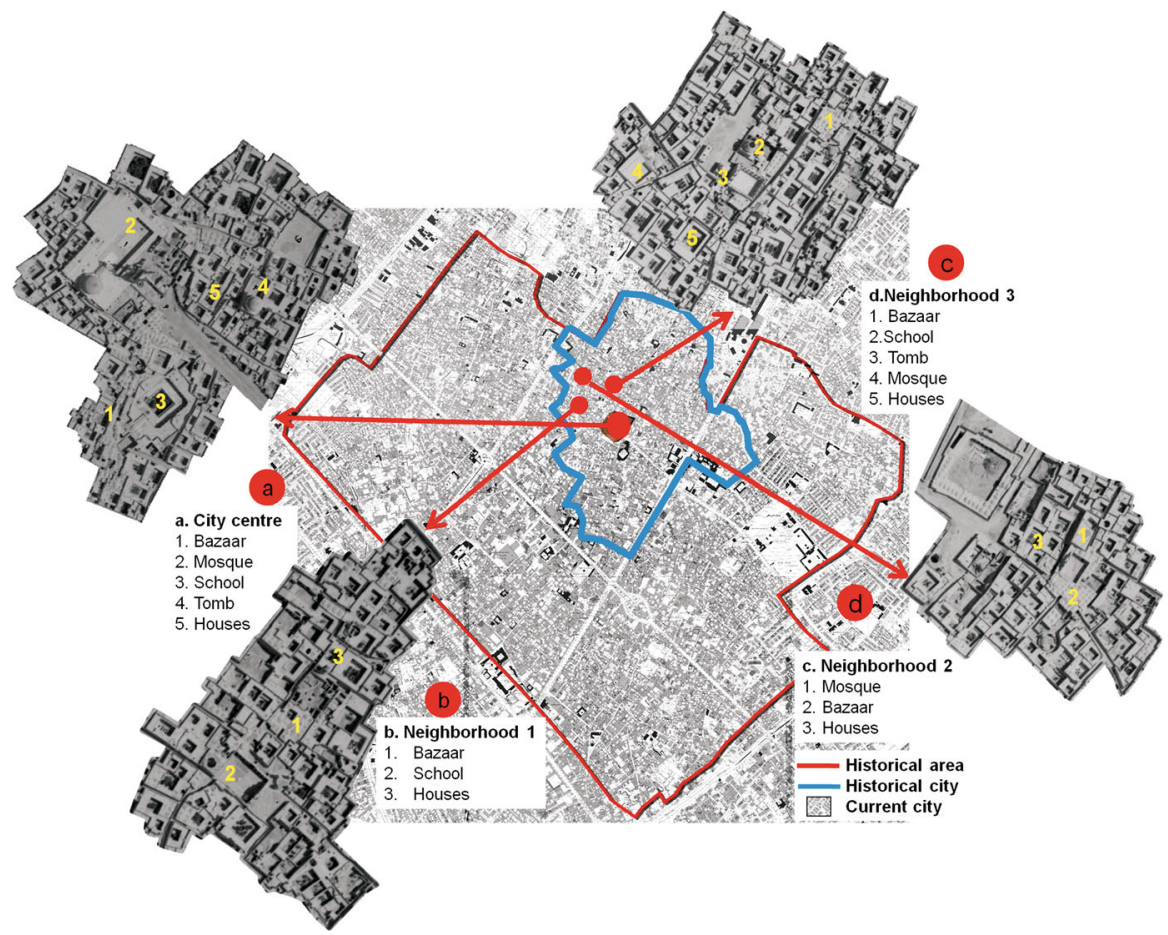

Fig. 3 The historical area (inside the red outline) and the historical city (inside the blue outline) of Yazd. The blue outline also marks the study area. a-d An aerial image of the city centre and three of its neighbourhoods showing the main structure of the historical city with different functions (Aerial images source: National Cartographic Centre of Iran 2005) 


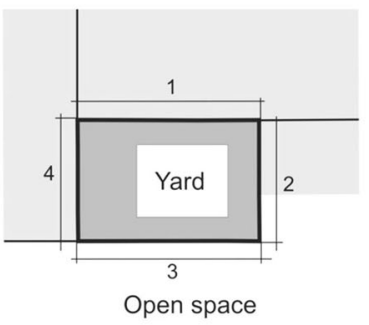

1

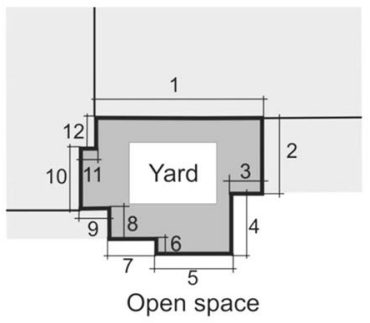

4

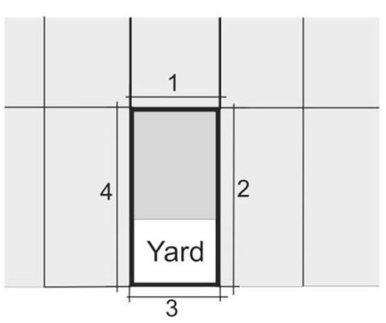

Open space

7

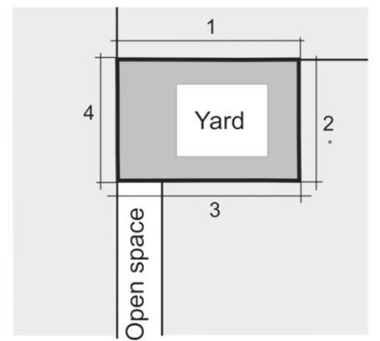

2

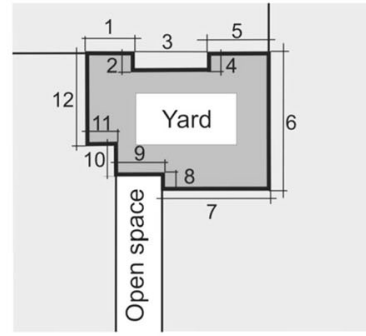

5

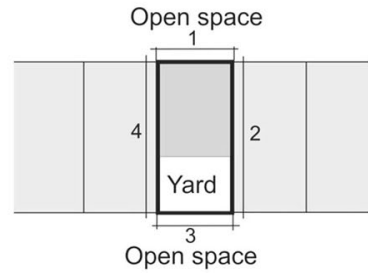

8

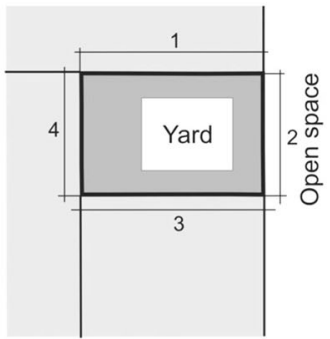

3

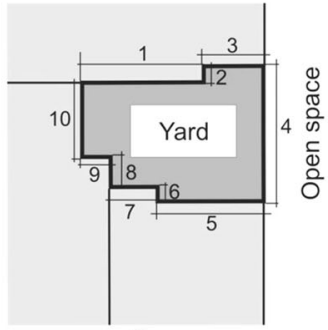

6

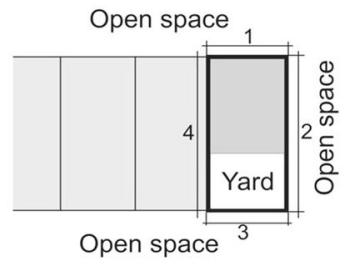

9

Fig. 4 Sketches of three typical spatial locations of courtyard houses in the old part (1-6) and houses in the new part (7-9). Sketches (1-3) show comparatively regular, and sketches (3-6) irregular, edge arrangements. The sketches also show how the edge lengths were measured and how the houses connect to nearby buildings and open spaces through regular and irregular edges. The open spaces can be squarelike $(1,4)$, paths/alleys $(2,5)$, and main paths $(3,6)$. Sketches (7-9) show the edge-length measurements of typical houses in the new part. The open spaces in the new part of the city are mainly streets

well as the edge lengths of courtyard houses. In addition, we measured the areas of many houses. Both the areas and edge lengths of ordinary houses were then compared with similar measurements of ordinary houses in the new part of the city.

The numbers of main structures measured in the historical part of the city are as follows: 4 bazaars, 5 mosques and tombs, and 3 schools. The numbers of edgelengths measured for these structures are 341 for bazaars, 302 for mosques and tombs, and 239 for schools. In the historical part we measured 6,804 edge-lengths of 1,243 courtyard houses and the same number $(1,243)$ of house areas. In the new part, we measured only ordinary houses, a total of 4,948 edge-lengths of 1,237 houses and the same number $(1,237)$ of house areas. 


\section{Measurement Methods}

Measurements of orientation, sizes of edge lengths, and areas of houses give rise to statistical frequency distributions that can be analysed in various ways. The orientation of the measured houses can be analysed through circular statistics and visualised by the rose diagrams (Fig. 5). We also use the technique of size distributions (Fig. 6) and entropy analysis of frequency distributions to obtain quantitative information on the differences in geometric patterns between the different parts of the city of Yazd.

\section{Circular Statistics and Rose Diagrams}

Circular statistics, which are primarily visualised by the rose diagrams, show the frequencies of data with different trends (Fig. 5). Rose diagrams provide a particularly clear visualisation of the variation in trend in two ways: as complete circles or as half circles (semi-circles). Rose diagrams are used to show the trends of either a certain process, such as the wind directions at a certain locality over a certain period (a wind rose), or the trends of certain lineaments such as fractures or
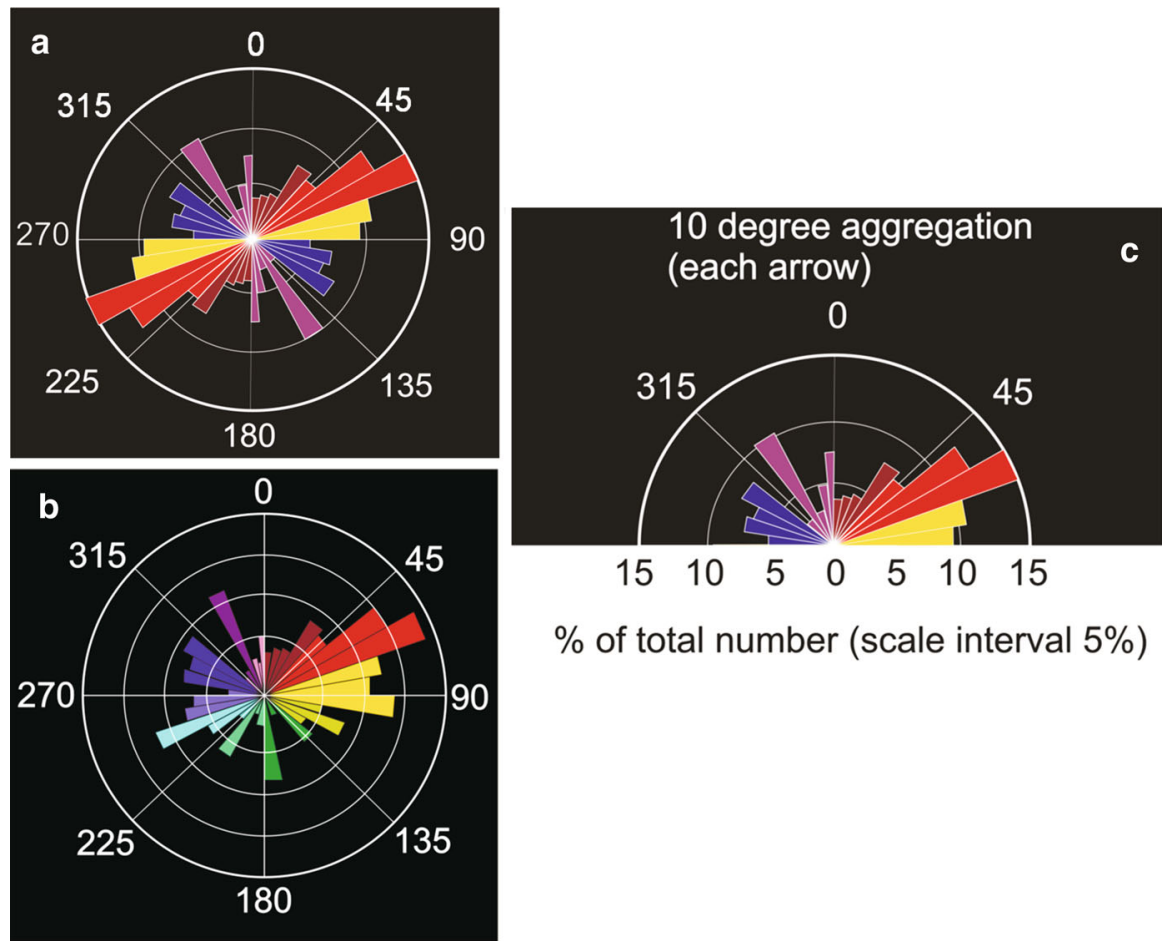

$\begin{array}{lllllll}15 & 10 & 5 & 0 & 5 & 10 & 15\end{array}$

$\%$ of total number (scale interval $5 \%$ )

Fig. 5 Simple rose diagrams showing the frequencies (numbers, percentages) of lineaments with different trends. a Rose diagram showing a bidirectional or symmetrical trend distribution, $\mathbf{b}$ rose diagram displaying a unidirectional or asymmetric trend distribution, and $\mathbf{c}$ northern half $\left(180^{\circ}\right)$ or semi-circle rose diagram (cf. Mohajeri et al. 2012) 

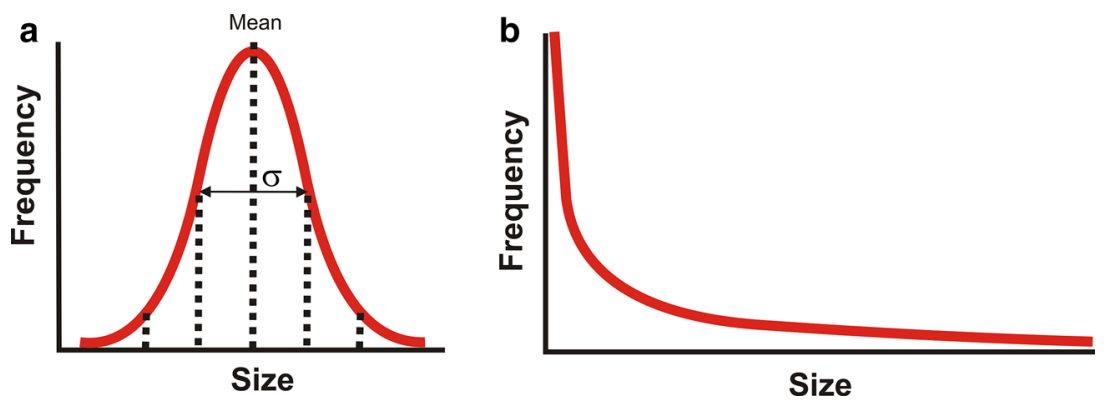

Fig. 6 a Normal distribution, with a standard deviation $(\sigma)$, and $\mathbf{b}$ a power-law (heavy-tail) size distribution

streets (Fisher 1993; Swan and Sandilands 1995; Smith et al. 2009). The present analysis of the edge-length trends uses the program GEOrient (http://www. holcombecoughlinoliver.com).

When analysing lineament trends, both directional and oriented data can be used. In directional data we can distinguish one end of the lineament from the other, or left from the right. This applies, for example, to the flow in a river or a dominating wind direction. Oriented data, by contrast, relate to phenomena without a directional distinction. These data include crustal fractures and streets in a city (Fisher 1993; Swan and Sandilands 1995). Thus, when the data are directional, then the rose diagram shows a unidirectional or asymmetric trend distribution (Fig. 5b). By contrast, when the data are oriented, then the rose diagram shows a bidirectional or symmetrical trend distribution (Fig. 5a). For directional data, the measured data azimuths range from $0^{\circ}$ to $360^{\circ}$. For oriented data, however, the opposite directions, $180^{\circ}$ apart, are equivalent. Thus, for oriented data, the graphical presentation should either be restricted to a semicircle (Fig. 5c) or have a rotational symmetry, in which case the opposite classes or sectors in the rose have the same frequency (Swan and Sandilands 1995). In this study, we use oriented data (edge lengths) so that the rose diagrams show bidirectional or symmetric trend distribution on a complete circle (Fig. 5a).

An additional point is that rose diagrams can be constructed using either normalised (weighted) or non-normalised (unweighted) data. The trends of houses are non-normalised when their edge lengths are not considered, in which case short edges and long edges have equal weight in the rose diagram. By contrast, when the edge trend is normalised by the length of the shortest edge, more weight is given to the long edges because they are then considered as composed of many short parts.

\section{Size Distributions}

Here we give a brief overview of normal distribution and power laws and their use in statistical size-distribution analyses. More details can be obtained from numerous books (e.g. Schroeder 1991; Liebovitch 1998; Peitgen et al. 2004; Brown and Liebovitch 2010).

The normal (Gaussian) distribution. The normal or bell-shaped distribution is widely regarded as the most important probability distribution in statistics (Fig. 6a; 
Ebdon 1985; Shaw and Wheeler 1985). In a normal distribution, the horizontal scale along the bottom of the distribution represents values of a variable, for example, the tallness (height) of people (Spiegel and Stephens 2011). The midpoint is the mean (arithmetic average) of the values. For a normal distribution, the data can be described in terms of the mean and the standard deviation $(\sigma)$, the latter being a measure of spreading. The normal-distribution mean, median (the middle value), and mode (the most common value) all coincide. A mixture of two normal distributions creates a bimodal frequency distribution or curve, with two different modes and means, which shows two distinct peaks or maxima (Spiegel and Stephens 2011).

Although normal distributions are very common, heavy-tailed power-law distributions are increasingly recognised as the fundamental statistical distributions in many physical, biological, social, economic, business and organisational phenomena (Newman 2005; Andriani and McKelvey 2009; Pisarenko and Rodkin 2010). In a normal distribution the variability of the sample data points are similarly distributed and generally clustered around the mean (Fig. 6a). Power-law distributions, by contrast, have many more data points with extreme values than the corresponding normal distribution. They thus exhibit a long or heavy tail (Fig. 6b). Normal distributions have a limited variance and stable means. Therefore, for a normal distribution, the data can be described through a typical value namely, the mean value. Power-law distributions have potentially infinite variance. When extreme events occur they also alter the value of the mean, pulling it towards the tail where the extreme events or object are located. For this reason, powerlaw means are unstable and this distribution has no typical size to describe the data (in contrast to the mean of a normal distribution). A power-law distribution implies that there are many small objects/processes and very few large ones.

The power-law size distribution. As indicated above, power-law size distributions are very common in artificial (man-made) and natural processes and structures (Fig. 6b). The populations of cities, the sizes (intensities) of earthquakes, word frequencies in literature, and the frequencies of family names all give rise to power-law distributions (e.g., Schroeder 1991; Peitgen et al. 2004; Newman 2005, 2010). As mentioned, power-law distributions imply that the number of small events, processes, or objects of a particular type is large in comparison with the number of large events, processes, or objects of the same type. The concept of a fractal, based on self-similarity, where the shapes or geometric patterns do not change when observed at different scales, reveals itself in a power law (Batty and Longley 1994; Bovill 1996; Liang et al. 2013). When applied to a cumulative frequency (probability) distribution, a power law has the form:

$$
P(\geq x)=C x^{-D}
$$

where $\mathrm{P}(\geq \mathrm{x})$ is here the number of edges (of a built structure such as a bazaar or a mosque), or the areas of houses larger than $\mathrm{x}, \mathrm{C}$ is a constant of proportionality, and $\mathrm{D}$ is the scaling exponent. To test if a size distribution is really a power-law, the normal procedure is to make bi-logarithmic $(\log -\log )$ plots, that is, to plot the $\operatorname{logarithms}$ of the value $(\mathrm{x})$ and its probability $\mathrm{P}(\mathrm{x})$, namely $\log (\mathrm{P}(\mathrm{x}))=\log (\mathrm{C})-$ $\mathrm{D} \log (\mathrm{x})$. If the $\log -\log$ plot gives a straight line, it is considered a power law. More accurate tests can also be made to compare the fit of the data to power laws in relation to other distributions; these tests are beyond the scope of the present paper, but the details are given by Clauset et al. (2009) and Mohajeri et al. (2013). 
In a power law, the straight line is, however, rarely observed over the entire range of the values or sizes of x. More commonly, there are one or more breaks in the log-log plot, yielding two or more straight lines with different slopes over different length ranges (values on the horizontal X-axis of the plot). These slopes are referred to as the scaling exponents of the power laws. When the log-log plot yields two such straight lines, the distribution is referred to as a double power-law (Newman 2005, 2010; Han et al. 2011).

\section{Entropy Analysis}

Entropy, normally denoted by the symbol $\mathrm{S}$, is a fundamental concept in thermodynamics and statistical mechanics, as well as in information theory and many other fields (Baierlein 1971; Kondepudi and Prigogine 1998; Nelson 2006; Desurvire 2009; Volkenstein 2009). In statistical mechanics and information theory it is demonstrated that entropy has a probability basis. The concept can therefore be used for analysing general frequency distributions, including the size distributions of edge-lengths. Then we use the following expression, known as Gibb's entropy formula in statistical mechanics and as Shannon's entropy in information theory (the only difference between these relates to the units of the constant $k$ ), which gives the entropy for a general probability distribution as:

$$
S=-k \sum_{i=1}^{t} p_{i} \ln p_{i}
$$

where $k$ is a constant, $\mathrm{t}$ is the number of classes or bins that contain edges or house areas in the frequency distribution, that is, the number of bins with nonzero probabilities of edge lengths/house areas, and $\mathrm{p}_{\mathrm{i}}$ is the frequency or probability of edges belonging to the i-th bin, that is, the probability of the i-th class or bin.

When we calculate the entropy using Eq. (3), only bins with at least one measured edge/area are included, that is, those bins where the probability of finding an edge/area is greater than zero. By definition, we also have that:

$$
\sum_{i=1}^{t} p_{i}=1
$$

meaning that the sum of the probabilities of all the bins is equal to one. Given that the probabilities are always between 0 and 1 (Eq. 3), and that the natural logarithms of numbers between 0 and 1 are negative, the minus sign in Eq. (2) is to ensure a positive value for the entropy (as it must be). The probabilities, as applied to edges/ areas in a population, are a measure of the chances of randomly selected edge/area from the population falling into a particular bin.

\section{Results}

Trend Analysis of Houses

Figure 3 shows the location of the main areas within the old part where the bazaars, mosques, tombs, and schools were measured. The figure also shows the connection 
of these structures with the surrounding houses through irregular edges and open spaces such as pathways, squares, and yards. The irregular shape of each structure, and the way the structures connect to each other and their surroundings, is partly due to the land divisions and ownerships and partly to the irregularity of the open spaces, mainly pathways (Memarian and Brown 2003).

Using the measured edge lengths, we show the orientation of the measured houses in the old and new parts in Fig. 7. Orientation is here defined as the trend of the longest edge. The total number of measured courtyard-house trends in the old part is 1,243, whereas the number of measured house trends in the new part is 1,237. The trends are shown by rose diagrams. Both weighted and unweighted edge-length trends are shown in Fig. 7.

The dominating trends of houses in the old and new parts are similar, mostly northeast-southwest and northwest-southeast, that is, roughly orthogonal. There is, however, a greater spread in the trend of houses in the old part. This follows because
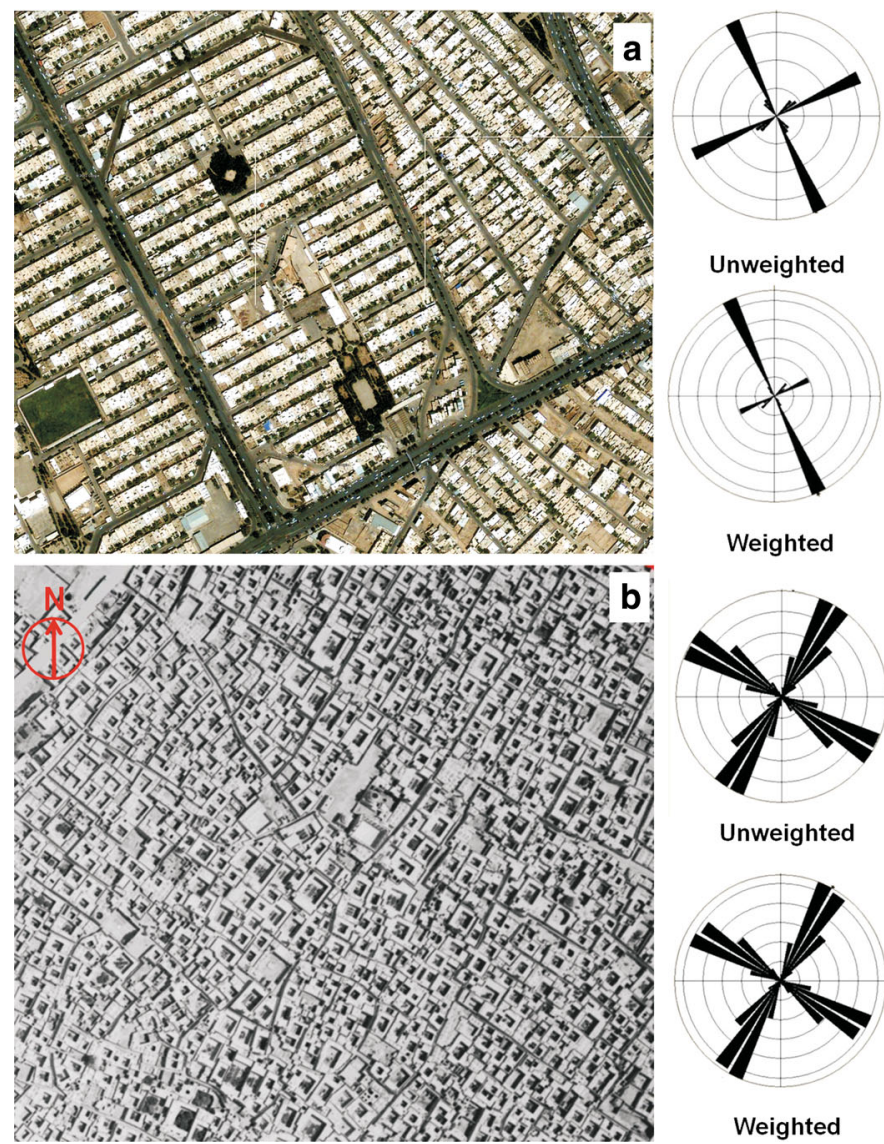

Weighted

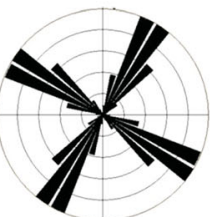

Unweighted

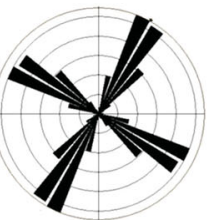

Weighted

Fig. 7 Rose diagrams (unweighted and weighted) showing the orientation of houses in (a) the new part of the city of Yazd (Google Earth), and (b) in the historical part of city (National Cartographic Centre of Iran 2005) 
the houses in the new part are associated with a strict grid-like street network, whereas in the old part the houses are associated with a street network which is more varied in geometry. It is likely that the trends reflect partly the climatic conditions, and partly the trends of the streets. It has been suggested that the dominating northeast-southwest trend, in particular, is well suited as regards the division of the houses into summer and winter quarters (Memarian and Brown 2003). Other factors that are likely to affect house trends include the pre-urban boundaries of fields, land divisions, and orchards. These lines (boundaries, division lines), in turn, are determined by the slope of lands and direction of water channels (Bonine 1979; Kostof 1991).

\section{Size Distributions of Edge-Lengths of Houses}

The edge lengths of the buildings in the old part are presented in Table 1. Standard regression methods are used to calculate the cumulative length-size power-law distributions in the ordinary plots (Figs. 8a, 9a) as well as in $\log -\log$ plots for the edge lengths (Fig. 8b, c, 9b). From the slopes of the straight lines on the log-log plots (e.g., Figs. 8c, 9b), we calculated the scaling exponents of the power laws.

The edge-length distributions are consistent with power laws that have different slopes for different edge-length ranges, that is, are double power laws (Figs. 8c, 9b). The breaks in the slopes of the edge-lengths occur at about $12 \mathrm{~m}$ for all the structures, except for mosques, where the break is at about $20 \mathrm{~m}$ (Table 1). The shallow-sloping straight lines range in scaling exponents (slopes) from 0.319 (houses) to 0.824 (bazaars), whereas the steep-sloping lines range in scaling exponents from 3.141 (schools) to 5.101 (mosques).

Table 1 Edge-length populations and subpopulations (A denotes shallow straight-line slopes, B denotes steep straight-line slopes, cf. Figs. 8, 9) for the old part of Yazd

\begin{tabular}{ccllll}
\hline Structures & Number of edges & Scaling exponent (D) & Entropy (S) & Length range & Range \\
\hline Bazaar & 341 & 2.219 & 2.253 & $0.41-48.52$ & 48.11 \\
A & 275 & 0.824 & 1.799 & $0.41-12$ & 11.59 \\
B & 66 & 3.783 & 2.207 & $12-48.52$ & 36.52 \\
Mosque & 302 & 1.673 & 2.617 & $0.31-48.52$ & 48.21 \\
A & 257 & 0.758 & 2.309 & $0.3-20$ & 19.69 \\
B & 45 & 5.101 & 2.164 & $20-48.52$ & 28.52 \\
School & 239 & 1.904 & 2.581 & $0.38-61$ & 60.62 \\
A & 163 & 0.501 & 1.876 & $0.38-12$ & 11.62 \\
B & 76 & 3.141 & 2.496 & $12-61$ & 49 \\
House & 6,804 & 1.989 & 2.622 & $1.5-125$ & 123.5 \\
A & 3,121 & 0.319 & 1.924 & $1.5-12$ & 10.5 \\
B & 3,683 & 3.488 & 2.464 & $12-125$ & 113 \\
\hline
\end{tabular}

The columns show the number of measured edge lengths, the scaling exponents (D), the entropies (S), the length ranges (the differences between maximum and the minimum edge lengths in a population) and the calculated length ranges 

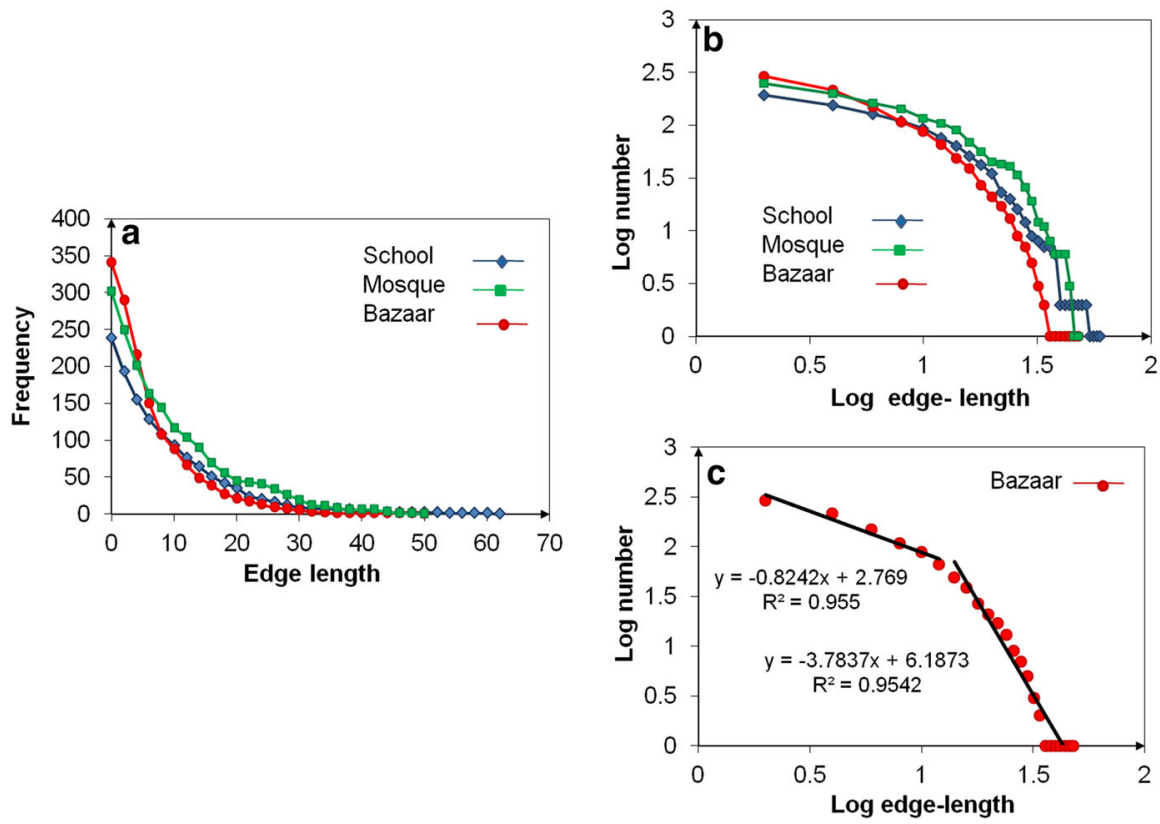

Fig. 8 Cumulative frequency edge-length size distribution for bazaars, mosques and tombs, and schools in the old part of the city. a Ordinary plot of edge lengths versus frequency. b Plot of log-transformed values. $\mathbf{c}$ Break in the straight-line slope at an edge-length of about $12 \mathrm{~m}$ for the bazaars
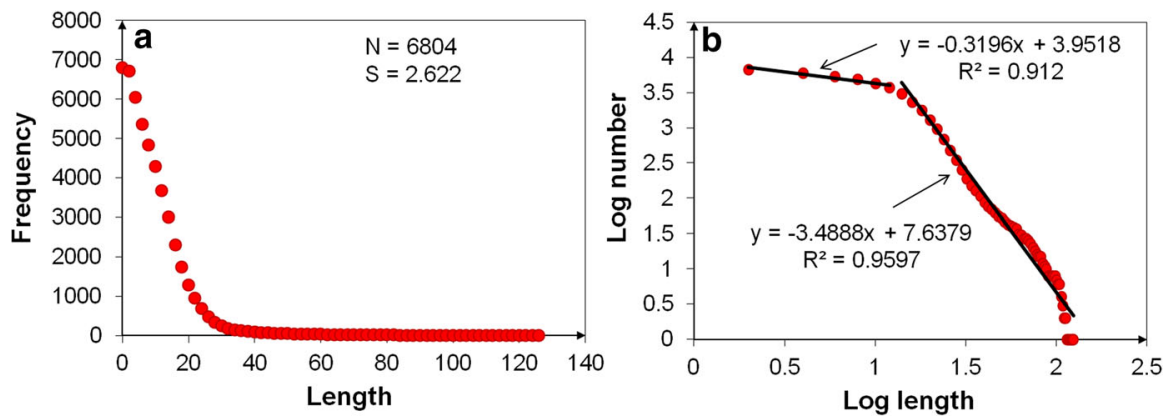

Fig. 9 Cumulative frequency edge-length distribution of houses $(6,805$ measurements $)$ from the old part of the city. a Ordinary values and b log-transformed values of the same data. Notice the break in the straight-line slope at an edge length of about $12 \mathrm{~m}$

Using the scaling exponents, each population can be divided into three (not all mutually exclusive) subpopulations, namely: (1) short edges (with shallow straightline slopes and low scaling exponents), (2) long edges (with steep straight-line slopes and high scaling exponents), and (3) the whole edge population for the specific structure. In Table 1, whole population is given its specific name (bazaar, mosque, etc.), and the subpopulation of short edges is denoted by $a$, and that of long 


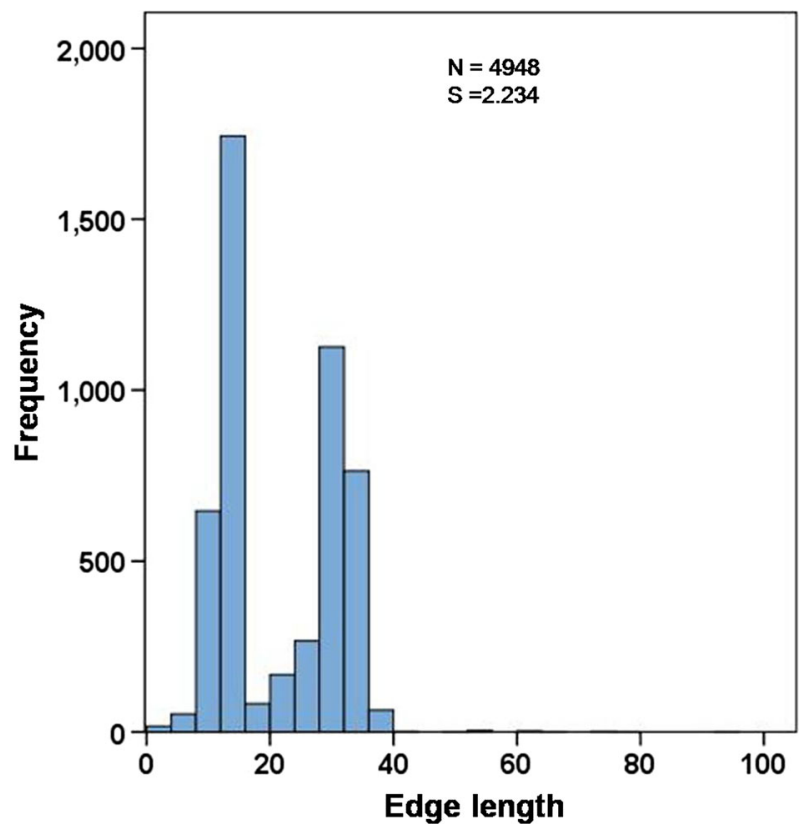

Fig. 10 Bimodal edge-length size distribution (4,948 measurements) from houses in the new part of the city

edges by $b$. For each of these populations, the length ranges and the entropies (Eq. 3) were calculated (Table 1).

For comparison with the old part, 4,949 edge lengths of 1,237 typical houses in the new part of the city were measured (Fig. 10). In contrast with the old part, the edge lengths of houses in the new part do not follow a power-law distribution but rather a bimodal distribution, that is, a distribution with two sharp peaks (Fig. 10). Commonly, such as here, a bimodal distribution is the result of a mixture of two (here roughly) normal distributions with different means and modes.

The bimodal distribution is easily explained for the edge lengths of the new part. Most of the measurements are from (mainly) rectangular buildings (Fig. 4) where the edge lengths form pairs. This means that the two edges that form the one pair are longer than the two edges that form the other pair. If the buildings are mostly similar in sizes (in square metres), as for the measured new part of the city of Yazd, there will be two peaks for the edge-length size distribution: one corresponding to the longer edges (or sides) of the buildings, the other to the shorter edges (or sides) of the buildings. The results also show that extremely long edges and extremely short edges are rare (Fig. 8). This follows because most of the buildings are similar in size; few are either very small or very large.

The entropy of the edge lengths of houses in the new part, $\mathrm{S}=2.234$ (Fig. 10), is less than that of the houses in the old part, $S=2.622$ (Fig. 9a). The results imply that, despite having a bimodal distribution, the edge lengths of houses in the new part are less dispersed than those in the old part of the city. The results also 
demonstrate quantitatively that there is a lack of coherence, in other words, a dissimilarity, in texture between the house structures of old and new parts of Yazd.

\section{Size Distributions of House Areas}

We also measured and compared the areas of 1,243 houses in the old part with 1,237 houses in the new part of the city. In the old part, the area-size distribution of houses follows a power law to a first approximation (Fig. 11), whereas in the new part it follows a bimodal distribution (Fig. 12). These results complement, and agree with, those for the edge-length size distributions for houses in the old (Fig. 9) and new (Fig. 10) parts.

The calculated entropies for the area-size distributions show very different results for the new and the old parts. The entropy for the areas of houses in the old part is $\mathrm{S}=2.638$ (Fig. 11c), whereas that of the areas of houses in the new part is $\mathrm{S}=1.629$ (Fig. 12b). Clearly, the entropy of the area-size distribution of houses in the old part is much higher than that of houses in the new part. The results fit well with the entropies calculated for the edge-length size distributions, although the entropy difference between the old and new part is larger for the area distribution than for the edge-length distribution.
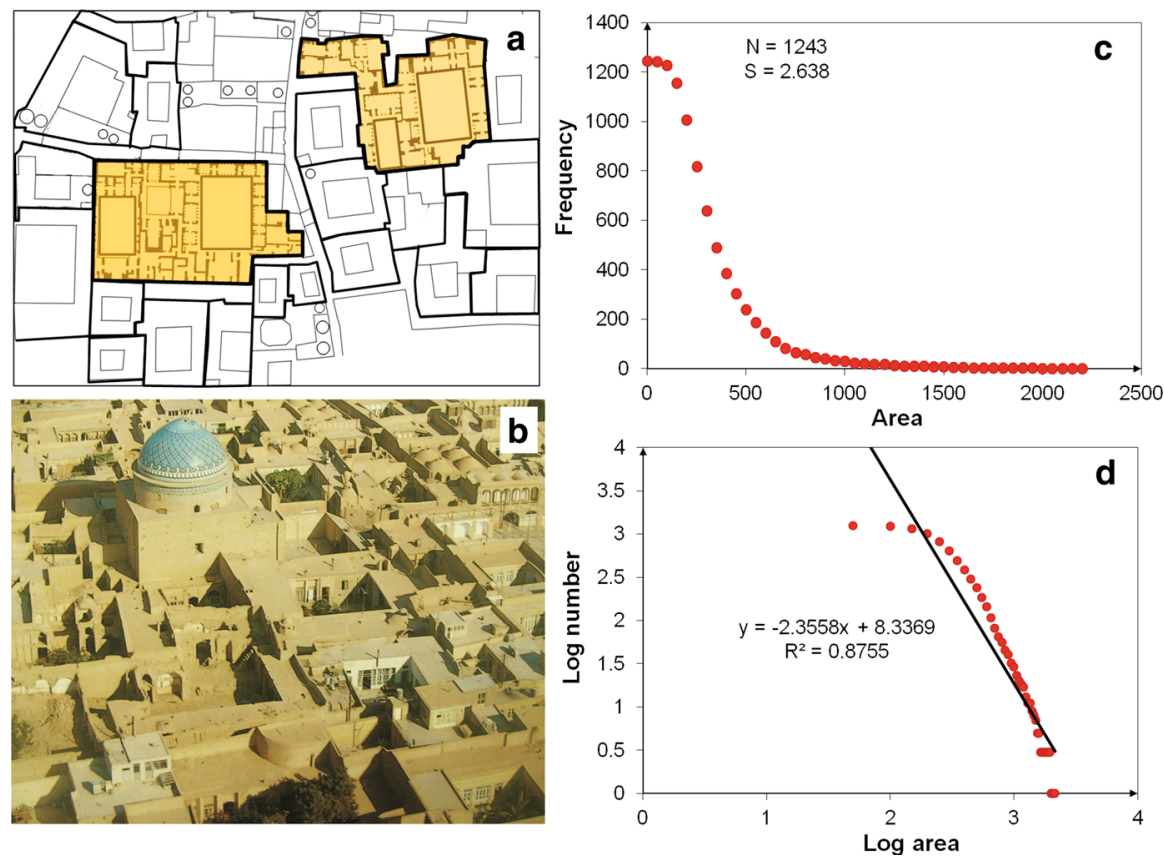

Fig. 11 a Sketch map of the historical part of Yazd (Tavasoli 2001) showing the measured area for a typical courtyard house based on the polygon footprints. b A birdseye-view of typical courtyard houses in the old part (Yazd Cultural Heritage and Tourism Organization 2005). c Ordinary plot and d logtransformed plot showing the power-law size distributions of house areas 

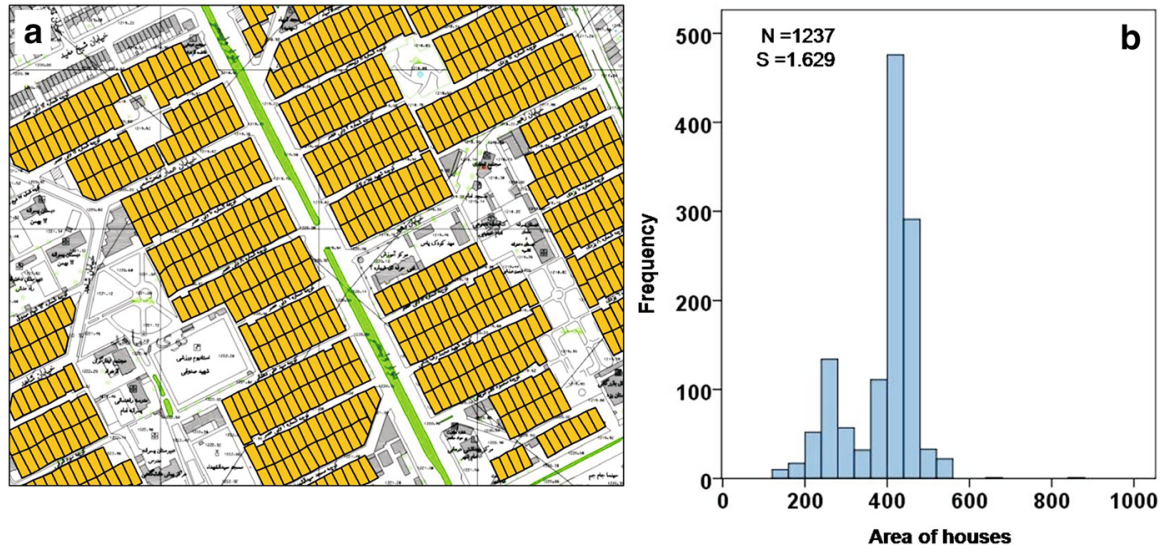

Fig. 12 a Part of a map of the new part of Yazd showing the areas of the houses as measured in this study, based on the polygon footprints. b Bimodal plot of house-area sizes

\section{Discussion and Conclusions}

The old parts of many Iranian cities consist of several basic structures including a mosque and tomb, a bazaar, a square, residential quarters, a religious school, and a public bathhouse (Habibi 1998; Tavasoli 2001; Karimi 2000; Sharifi and Murayama 2013). All these physical structures are multifunctional and have important characteristics such as socio-economic, cultural, environmental, and political (Clark and Costello 1973; Kheirabadi 2000). In particular, they have been, to a large extent, successful in accommodating social and environmental needs (Kasmaii 1983; Tavasoli 2001; Soflaee and Shokouhian 2005; Mobaraki et al. 2012; Sharifi and Murayama 2013). The fine integration and cohesion of the physical structures in the old part of many Iranian cities is one of their most prominent characteristics (Tavasoli 2001; Sharifi and Murayama 2013).

However, the new developments of the city structures often seem to lack the detailed integration and cohesion of the old part-and this lack may be partly the result of modernisation during the last century (Habibi 1998; Karimi 2000; Fanni 2006; Sharifi and Murayama 2013). Many of the old city structures mentioned above lost some of their characteristics as well as their functionality and gradually became parts of much larger cities with poor connection with the new parts. The old and new parts of cities are to a degree almost separated and are poorly connected. In addition, the physical characteristics as well as the social and environmental characteristics of the old and new parts are commonly totally different (Tavasoli 2001; Sharifi and Murayama 2013). In this study we have shown quantitatively some differences between the physical characteristics of the old and the new (most recent) parts, focusing on the bazaar, mosque, school from the old part, and residential quarters in both old and new parts. Other aspects of these differences could be explored in the future when more data is available.

A comparison between the edge-length size distributions in the old part and new parts shows a striking difference. In the old part, the distributions follow power laws 
for all the structures studied here (bazaars, mosques, and schools) including courtyard houses (Figs. 8,9). By contrast, the edge lengths of houses in the new part follow a bimodal distribution (Fig. 10), apparently the result of the mixture of two (roughly) normal distributions. Generally, structures in the old part are irregular, that is, have uneven edge lengths (Fig. 4), which makes them highly interconnected and coherent. The high degree of interconnectivity at the small scale is also reflected in the coherence of the overall structure of the old part of the city at a large scale (Figs. 2, 3, 4). The edges in the newer parts are regular and less interconnected in a statistical sense (Fig. 7) than in the old part.

The area-size distributions of the houses in the old and the new part of the city yield similar results to those of the edge-length size distributions. The areas in the old part show power-law size distribution (Fig. 11), whereas those in the new part show bimodal size distribution (Fig. 12).

The calculated entropies of the size-frequency distributions, both for edge lengths and for house areas, also show significant differences between the old and the new parts. For the edge lengths, the entropies are 2.622 (old part) and 2.234 (new part), whereas for the house areas the results are 2.638 (old part) and 1.629 (new part). The edge lengths and house areas in the old part follow power laws (Figs. 8, 9, 11) that yield greater dispersal or spreading, and thus higher entropies (Baierlein 1971; Blundell and Blundell 2006; Kardar 2007), than the bimodal distributions in the new part.

Since length range is the difference between the maximum and the minimum edge length in each population, a larger length range implies a longer tail of the power-law distribution which may be reflected in larger entropy (a larger dispersion or spread in the data). To test this, we plotted entropy versus length range for the old (power-law) part (Fig. 13). When the courtyard houses are included (Fig. 13a) coefficient of determination is $\mathrm{R}^{2}=0.552$, but $\mathrm{R}^{2}=0.750$ when the houses are omitted from the data set (Fig. 13b). We decided to use the latter also because the number of measured houses is so many times greater than those of measured bazaars, mosques, tombs, and schools combined and thus dominates the correlation determination. Generally, the linear correlations mean that as the length range increases, so does the entropy of the population.
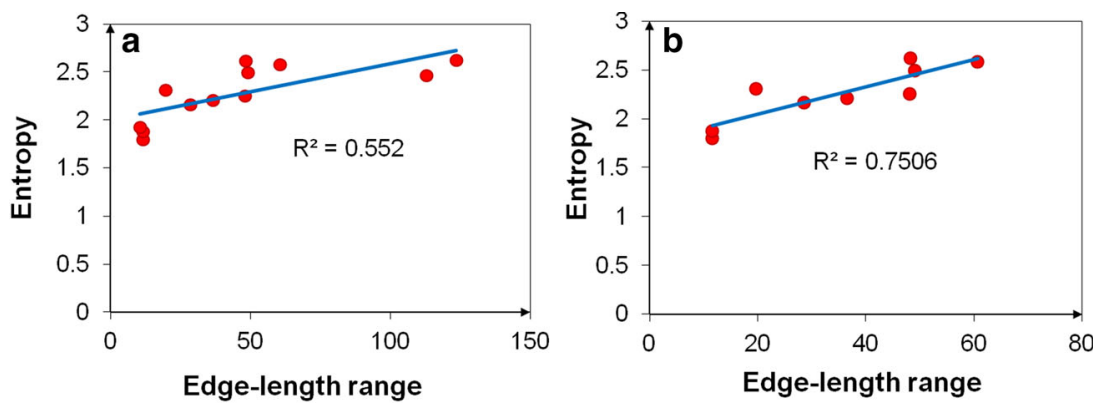

Fig. 13 a Entropy plotted against the range in edge length of the populations and subpopulations in Table 1, including courtyard houses. b Entropy plotted against the range in edge length with the courtyard houses are excluded 
The results show a clear geometric and quantified (through edge-length size and house-area size distributions) differences between the old and the new parts of Yazd. These methods, presented here (to the best of our knowledge) for the first time, should be very useful in analysing and quantifying the urban morphology and the geometric patterns in cities in general. They should be particularly useful for studying those cities that have evolved over long periods of time and contain new parts that have evolved largely independently of the older parts.

The present results show that, perhaps in contrast to what we could have expected, the edge lengths and house areas in the old part of the city of Yazd show clear power-law size distributions. By contrast, the edge lengths and house areas in the new part of the city show bimodal size distributions. The power-law distributions indicate a gradual and continuous change in sizes of edge lengths and house areas based in the old part of city, whereas bimodal distributions indicate abrupt (discontinuous) changes in the edge lengths and house areas in the new part. This gradual change in the old part may be regarded as more natural, as indicated by the power-law size distributions of many landscape forms and lineaments (Turcotte 1997). One suggested implication of the results presented here is that the structures in the old part of Yazd are well interconnected, form a coherent whole, and provide a quantitative measure of the clear geometric difference between the old and the new parts of the city.

The present new methods for analysing the geometric differences between new and old parts of cities have, for the case study of Yazd, shown clear textural differences between the old and new part of the city. Since these methods are completely general, they should be applicable to similar analyses worldwide and be particularly suitable for the analysis of fast-growing small to medium-size cities. One development of the methods presented here is to measure different quantities, such as the heights or tallness of the houses, and relate them to the edge lengths and areas of the houses. Also, further expansion of the analysis presented here could, in addition to quantifying the physical differences, include the socio-economic and environmental differences between the old and new parts of cities. These aspects of the present methods might suggest solutions for making the old and new (more recent) parts better integrated and are worth exploring in future studies.

The methods presented here can also be used to make quantitative studies of different cities, in different environments, and relate the results to energy considerations and general building physics. Such a development should provide results of great value for authorities and decision makers on environmentally sustainable policies regarding urban design and planning.

\section{References}

Andriani, P., and B. Mckelvey. 2009. From Gaussian to paretian thinking: causes and implications of power laws in organizations. Organization Science 20:1053-1071.

Baierlein, R. 1971. Atoms and information theory: an introduction to statistical mechanics. San Francisco: W. H. Freeman.

Batty, M., R. Carvalho, A. Hudson-Smith, R. Milton, D. Smith, and P. Steadman. 2008. Scaling and allometry in the building geometries of greater London. The European Physical Journal B 63:303-314. 
Batty, M., and P. Longley. 1994. Fractal Cities. London: Academic Press.

Blundell, S.J., and K.M. Blundell. 2006. Concepts in thermal physics. Oxford: Oxford University Press.

Bonine, M.E. 1979. The morphogenesis of Iranian cities. Annals of the Association of American Geographers 69:208-224.

Bovill, C. 1996. Fractal geometry in architecture and design. Boston: Birkhauser Verlag.

Brown, C., and L.S. Liebovitch. 2010. Fractal analysis. London: SAGE Publications.

Clauset, A., R.C. Chalizi, and M.E.J. Newman. 2009. Power-law distributions in empirical data. Society for Industrial and Applied Mathematics 51:661-703.

Clark, B.D., and V. Costello. 1973. The urban system and social patterns in Iranian cities. Transactions of the Institute of British Geographers 59:99-128.

Desurvire, E. 2009. Classical and quantum information theory. Cambridge: Cambridge University Press.

Ebdon, D. 1985. Statistics in geography, 2nd ed. Oxford: Blackwell.

English, P.W. 1998. Qanats and lifeworlds in Iranian Plateau villages. Transformation of Middle Eastern Natural Environments 10:187-205.

Fanni, Z. 2006. Cities and urbanization in Iran after the Islamic revolution. Cities 23:407-411.

Fisher, N.I. 1993. Statistical analysis of circular data. Cambridge: Cambridge University Press.

Fuller, R.J., and R.H. Crawford. 2011. Impact of past and future residential housing development patterns on energy demand and related emissions. Journal of Housing and the Built Environment 26:165-183.

Gudmundsson, A., and N. Mohajeri. 2013. Entropy and order in urban street networks. Scientific Reports 3:3324. doi:10.1038/srep03324.

Habib, F., I. Etesam, H. Ghoddusifar, and N. Mohajeri. 2012. Correspondence analysis: a new method for analyzing qualitative data in architecture. Nexus Network Journal 14:517-538.

Habibi, S.M. 1998. Az Shar ta Shahr: Tahlili tarikhi az mafhume shahr va simaye kalbodiean, tafakor va tasvor. Iran: University of Tehran.

Han, D.D., J.H. Qian, and Y.G. Ma. 2011. Emergence of double scaling law in complex systems. Europhysics Letters 94:28006.

Hu, X. 2008. Boundaries and openings: spatial strategies in the Chinese dwelling. Journal of Housing and the Built Environment 4:353-366.

Iranian Statistical Centre. 2009. Census data. Iran: Tehran Statistical Centre.

Johansson, E. 2006. Influence of urban geometry on outdoor thermal comfort in a hot dry climate: a study in Fez, Morocco. Building and Environment 41:1326-1338.

Kalantari, H., and H. Hataminejad. 2006. The Rehabilitation Planning of the Traditional Part of Yazd city. Iran (in Persian): Faragoshtar Press.

Kardar, M. 2007. Statistical physics of particles. Cambridge: Cambridge University Press.

Kämpf, J.H., M. Montavon, J. Bunyesc, R. Bolliger, and D. Robinson. 2009. Optimisation of buildings' solar irradiation availability. Solar Energy 84:596-603.

Karimi, K. 2000. Urban conservation and spatial transformation: preserving the fragments or maintaining the 'spatial spirit'. Urban Design International 5:221-231.

Kasmaii, M. 1983. Climate and Architecture. Iran: Tehran Sherkat Khanesazi-Iran (In Persian).

Kheirabadi, M. 2000. Iranian Cities: formation and development. New York: Syracuse University Press.

Kondepudi, D., and I. Prigogine. 1998. Modern thermodynamics. New York: Wiley.

Kostof, S. 1991. The city shaped. London: Thames and Hudson.

Lambton, A.K.S. 1992. The qanats of Yazd. Journal of the Royal Asiatic Society of Great Britain and Ireland 2:21-35.

Lampl, P. 1968. Cities and planning in the ancient near east (planning and cities). New York: George Braziller Inc.

Lavas, G.P. 1974. Settlements in ancient Greece. Ekistics-the Problems and Science of Human Settlements 38:330-335.

Liang, J., Y. Hu, and H. Sun. 2013. The design evaluation of the green space layout of urban squares based on fractal theory. Nexus Network Journal 15:33-49.

Liebovitch, L.S. 1998. Fractals and chaos simplified for the life sciences. Oxford: Oxford University Press.

Marshall, S. 2005. Streets and patterns. London: Taylor and Francis.

Memarian, G., and F.E. Brown. 2003. Climatic, culture, and religion: aspects of the traditional courtyard house in Iran. Journal of Architectural and Planning Research 20:181-198.

Ministry of Housing and Urban Development. 2005. Master plan of Yazd. Iran (Tehran): Centre Information Iran. 
Mobaraki, O., J. Mohammadi and A. Zarabi. 2012. Urban form and sustainable development: The case of Urmia city. Journal of Geography and Geology, 4. doi:10.5539/jgg.v4n2p1.

Mohajeri, N., P. Longley, and M. Batty. 2012. City shape and the fractality of street patterns. Quaestiones Geographicae 31:29-37.

Mohajeri, N., and A. Gudmundsson. 2012. Entropies and scaling exponents of street and fracture networks. Entropy 14:800-833.

Mohajeri, N., J. French, and M. Batty. 2013. Evolution and entropy in the organisation of urban street patterns. Annals of GIS 19:1-16.

National Cartographic Centre of Iran. 2005. Aerial Images of Yazd. Iran: Tehran Cartographic Centre.

Nelson, P. 2006. Biological physics: energy, information, life. New York: W.H. Freeman.

Newman, M.E.J. 2005. Power laws, Pareto distributions and Zipf's law. Contemporary Physics 46:323-351.

Newman, M.E.J. 2010. Networks: an introduction. Oxford: Oxford University Press.

Peitgen, H.O., H. Jurgens, and D. Saupe. 2004. Chaos and fractals, 2nd ed. New York: Springer.

Pisarenko, V., and M. Rodkin. 2010. Heavy-tailed distributions in disaster analysis. Heidelberg: Springer.

Ratti, C., D. Raydan, and K. Steemers. 2003. Building form and environmental performance: archetypes, analysis and an arid climate. Energy and Building 35:49-59.

Salingaros, N.A., and B.J. West. 1999. A universal rule for the distribution of sizes. Environment and Planning B-Planning and Design 26:909-923.

Salingaros, N.A. 2005. Principles of urban structure. The Netherlands: Techne Press.

Scellato, S., A. Cardillo, V. Latora, and S. Porta. 2006. The backbone of a city. European Physics Journal B 50:221-225.

Schroeder, M. 1991. Fractals, chaos, power laws. New York: W.H. Freeman.

Sharifi, A., and A. Murayama. 2013. Changes in the traditional urban form and the social sustainability of contemporary cities: a case study of Iranian cities. Habitat International 38:126-134.

Shaw, G., and D. Wheeler. 1985. Statistical techniques in geographical analysis. New York: Wiley.

Smith, M.J., M.F. Goodchild, and P. Longley. 2009. Geospatial analysis: a comprehensive guide, 3rd ed. London: Troubador Publishing Ltd.

Soflaee, F. and M. Shokouhian. 2005. Natural cooling systems in sustainable traditional architecture of Iran. International Conference on Passive and Low Energy Cooling for the Built Environment, May 2005, Santorini.

Spiegel, M., and L.J. Stephens. 2011. Statistics. USA: McGraw-Hill.

Steemers, K., N. Baker, D. Crowther, J. Dubiel, and M. Nikolopoulou. 1998. Radiation absorption and urban texture. Building Research and Information 26:103-112.

Swan, A.R.H., and M. Sandilands. 1995. Introduction to geological data analysis. Oxford: Blackwell.

Tavasoli, M. 1989. Urban design in the central part of Yazd city. Tehran (in Persian): A Centre of Urban Research Press.

Tavasoli, M. 2001. The structure of city in hot-arid areas of Iran. Tehran (in Persian): Payam and Peivand Press.

Turcotte, D.L. 1997. Fractals and chaos in geology and geophysics, 2nd ed. Cambridge: Cambridge University Press.

Volkenstein, M.V. 2009. Entropy and information. Berlin: Birkhauser.

Yazd Cultural Heritage and Tourism Organization. 2005. Library Archives. Iran: Yazd Library archives.

Zanganeh Shahraki, S., D. Sauri, P. Serra, S. Modugno, F. Seifoddini, and A. Pourahmad. 2011. Urban sprawl pattern and land-use change detection in Yazd, Iran. Habitat International 35:521-528.

Nahid Mohajeri is an Architect, Urban Designer, and Geographer. She is currently a postdoctoral fellow at the Swiss Federal Institute of Technology in Lausanne (EPFL), Solar Energy and Building Physics Laboratory. Her main research interests are in physics of urban form, including urban complex systems and statistical modelling of urban patterns.

Agust Gudmundsson is a Professor who holds a University of London Chair of Structural Geology at Royal Holloway. His main research interests are in the physics of various processes and structures, including complex networks. He is the author of Rock Fractures in Geological Processes, published by Cambridge University Press (2011). 